\title{
A comparative assessment of two different debris flow propagation approaches - blind simulations on a real debris flow event
}

L. M. Stancanelli and E. Foti

Correspondence to: L. M. Stancanelli (lmstanca@dica.unict.it) 
